\title{
Cyclophosphamide inhibits the progression of Meniere's disease by reducing the generation of circulating immune complex
}

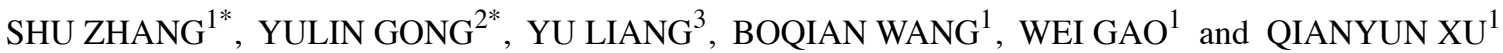 \\ Departments of ${ }^{1}$ Otolaryngology and ${ }^{2}$ Rheumatology and Immunology, \\ ${ }^{3}$ Computer Information and Network Management Center, The Affiliated Hospital of \\ Inner Mongolia Medical University, Hohhot, Inner Mongolia 010110, P.R. China
}

Received July 20, 2020; Accepted November 26, 2020

DOI: $10.3892 /$ etm.2021.10611

\begin{abstract}
Endolymphatic hydrops is a characteristic pathological manifestation of Meniere's disease (MD) that has been previously associated with autoimmunity. Interest in the circulating immune complex (CIC) has increased due to its reported role in the occurrence of MD. The present study aimed to investigate the potential value of serum CIC concentration in the diagnosis of MD and the therapeutic potential of cyclophosphamide (CTX) for the treatment of MD. In the present study, guinea pigs were immunized with isologous crude inner ear antigens to establish an autoimmune MD model. Pure tone audiometry, Vestibular-evoked myogenic potential test, electrocochleography test and auditory brainstem response was applied in this study for assessing the severity of MD in guinea pigs. ELISA was applied to measure CIC, tumor necrosis factor $\alpha$ (TNF- $\alpha$ ) and heat shock protein 70 (HSP70) expression levels in the serum samples of different groups of patients. Western blotting was applied to detect the protein expression of HSP70 in inner ear tissues in guinea pigs. Hematoxylin and eosin staining was applied to visualize the spiral ganglions in spiral ganglions models. CIC expression in the inner ear was detected by immunohistochemistry. In vivo experiments were performed to confirm the therapeutic effects of CTX in MD. Serum
\end{abstract}

Correspondence to: Dr Shu Zhang, Department of Otolaryngology, The Affiliated Hospital of Inner Mongolia Medical University, 1 Tongdao North Street, Hohhot, Inner Mongolia 010110, P.R. China E-mail:kuzhle@163.com

*Contributed equally

Abbreviations: ABR, auditory brainstem response; CIC, circulating immune complex; CTX, cyclophosphamide; ECochG, electrocochleogram; HSP70, heat shock protein 70; ICIEAg, isologous crude inner ear antigens; MD, Meniere's disease; TNF- $\alpha$, tumor necrosis factor- $\alpha$; VEMP, vestibular evoked myogenic potential

Key words: circulating immune complex, cyclophosphamide, heat shock protein 70, Meniere's disease, tumor necrosis factor- $\alpha$ concentrations of CIC, TNF- $\alpha$ and HSP70 were found to be significantly higher in patients with MD, which were also associated with increases in hearing classification and the severity of endolymphatic hydrops. Using a guinea pig MD model, ELISA results revealed significantly increased serum CIC, TNF- $\alpha$ and HSP70 concentrations compared with those in the control group. ABR results showed that the thresholds in the CTX group were notably decreased compared with that in the dexamethasone group, whereas CIC concentrations in the serum were reduced following dexamethasone and CTX treatments compared with those after saline treatment. In the inner ear tissues, the CIC concentration in CTX group was lower than that in the dexamethasone group. Similarly, reductions in HSP70 and TNF- $\alpha$ concentrations was also observed in a similar manner. Immunohistochemistry staining found notably lower CIC deposition in the inner ear tissues following CTX treatment than that in dexamethasone group. Taken together, higher CIC expression can be used as a biomarker for MD diagnosis. The efficacy of CTX in MD was found to be higher compared with that in dexamethasone, which may be associated with the effective inhibition of CIC, HSP70 and TNF- $\alpha$ generation.

\section{Introduction}

Meniere's disease (MD) is a complex inner ear disease that clinically manifests as recurrent rotatory vertigo, sensorineural hearing loss, tinnitus and sensation of ear fullness (1). Previous epidemiological studies found that there were 17-513 patients with MD per 100,000 individuals in Asia, where prevalence is markedly higher compared with that for systemic lupus erythematosus and multiple lupus erythematosus $(2,3)$. Endolymphatic hydrops is a characteristic pathological manifestation of MD (4). Although a previous study has found that the etiology of endolymphatic hydrops may be associated with autoimmunity, allergy, inheritance and infection, there is no definitive cause to this condition (5). Since the etiology and pathogenesis of MD remain unclear, no specific diagnostic guidelines are available that can be used in clinical settings (6,7). In addition, empirical symptomatic or surgical treatments and drug therapy for treating the mechanism of action of MD are also unavailable $(6,7)$. Therefore, it is important and of scientific significance to investigate the 
pathogenesis, potential auxiliary diagnostic marker(s) and therapeutic agents for MD.

Immunological factors are important in the development of MD in some patients (8). A previous study demonstrated that the endolymphatic sac is important in immune responses, where they can mediate the development of endolymphatic hydrops (9). MD may be an immune-mediated disease or even an autoimmune disease (10). In a previous study investigating the immunological mechanism of MD, Harada et al (11) used stria vascularis tissues from rabbits as antigens to immunize guinea pigs to construct an endolymphatic hydrops model. Immunoglobulin $\mathrm{G}$ antibody and complement components were detected in the endolymph fluid of the experimental guinea pigs, leading to the hypothesis that circulating immune complex (CIC) deposited in the stria vascularis of the inner ear damages the secretion and absorptive function of the endolymph fluid, resulting in endolymphatic hydrops (11). Numerous studies have also demonstrated that the CIC level was significantly higher in most patients with MD (12-14). Therefore, the role of the immune complex during the onset of MD has become an interesting topic of research. However, there remains to be a lack of investigations into the association between CIC levels in patients with MD and the severity, clinical stages and prognosis assessment of endolymphatic hydrops.

MD remains to be a disease that is difficult to diagnose, particularly in the early stages due to the absence of significant symptoms. Therefore, for complex inner ear diseases, such as MD, the therapeutic goals should not be limited to only controlling the symptoms. Instead, it is important to also develop tools for the effective diagnosis and treatment of inner ear diseases. Of note, there is a trend to specifically select a treatment to block the generation of endolymphatic hydrops by analyzing the causes of endolymphatic hydrops (15). Immunotherapy have been widely used for the treatment of tumors $(16,17)$ and have been documented to be beneficial in controlling the symptoms in patients with MD $(18,19)$. However, to the best of our knowledge, no previous study exist that clearly addressed the mechanism of action and efficacy of immunosuppressants in MD. Cyclophosphamide (CTX) is a nitrogen mustard derivative that is hydrolyzed by phosphoamidase or phosphatase activities in the liver or tumors to become the phosphoramide nitrogen mustard (20). CTX is toxic to rapidly proliferating cells and mediate their effects primarily by weakening DNA synthesis, rendering it to be a commonly used immunosuppressant (21). In addition, CTX has been widely used for numerous different diseases, such as acute leukemia, lymphoma, solid tumors and several autoimmune diseases, including severe rheumatoid arthritis, systemic lupus erythematosus, nephrotic syndrome in children and multiple granuloma $(22,23)$. However, studies investigating the efficacy of CTX for the treatment of MD remain insufficient.

Therefore, the present study aimed to explore the diagnostic value of serum CIC in MD patients, in addition to investigating the therapeutic potential of CTX for the treatment of MD. Data on patients with MD in a clinical setting was collected in the present study, following which CIC expression levels in different clinical stages of MD were statistically analyzed. Additionally, the potential value of using CIC for the diagnosis and treatment of patients with MD was also investigated. In another branch of the present study, an autoimmune MD guinea pig model was constructed to compare the association between CIC change prior to and following CTX treatment and the degree of endolymphatic hydrops and clinical symptom improvement to investigate the therapeutic potential in MD.

\section{Materials and methods}

Clinical sample collection. A total of 48 unilateral patients with MD (20 males and 28 females; mean age, $43.79 \pm 1.35$ years) who were diagnosed between July 2017 and May 2019 were collected and screened based on the Diagnosis and Treatment Guidelines of Meniere's Disease (2017) (24) from The Affiliated Hospital of Inner Mongolia Medical University (Hohhot, China). Patients with nephritis, systemic lupus erythematosus, allergic diseases and other immune system diseases and those who received hormone or immunosuppressive therapy within the last 3 months were excluded. In addition, another 48 age-matched healthy adult individuals (18 males and 30 females; mean age, $45.27 \pm 1.22$ years), who came in for physical examination at The Affiliated Hospital of Inner Mongolia Medical University, were recruited as the control group. All individuals were fasted for $6 \mathrm{~h}$ before the collection of $10 \mathrm{ml}$ peripheral venous blood, which was subsequently stored at $-20^{\circ} \mathrm{C}$ for subsequent experiments. The present study was reviewed and approved by the Ethics Committee of The Affiliated Hospital of Inner Mongolia Medical University and written informed consent was provided from the patients and the healthy controls.

Clinical diagnostic criteria. The following clinical diagnostic criteria for MD was used: i) In total, > two vertigo onsets, each lasting between $20 \mathrm{~min}$ and $12 \mathrm{~h}$; ii) in total, $\geq$ one audiological examination confirming low to medium sensorineural hearing loss of the affected ear during the course of the disease; and iii) the affected ear had fluctuating hearing loss, tinnitus and/or sensation of ear fullness. The following exclusion criteria were used: i) Vertigo induced by other diseases, including vestibular migraine, sudden deafness, benign paroxysmal positional vertigo, labyrinthitis, vestibular neuronitis, paroxysmal vestibulopathy, drug toxic vertigo, posterior circulation ischemia and intracranial space-occupying lesion; and ii) secondary endolymphatic hydrops.

Pure tone audiometry and disease classification method. A pure tone audiometer (GSI 61 ${ }^{\mathrm{TM}}$; Grason-Stadler, Inc.) was used for pure tone audiometry. The average air conduction thresholds at $0.5,1,2$ and $4 \mathrm{kHz}$ were obtained from the result of the auditory threshold. In addition, disease was classified based on the following classes from the result of pure tone audiometry (25): i) Class 1 , pure tone average (PTA) $<25$ decibels hearing level (dBHL); ii) Class 2, PTA=26-40 dBHL; iii) Class 3, PTA=41-70 dBHL; and iv) Class 4, PTA >70 dBHL.

Magnetic resonance imaging (MRI). The mix of normal saline and gadopentetate dimeglunmine $(0.5 \mathrm{ml} /$ person $)$ injection (Consun Pharmaceutical Group, Ltd.) was used as the contrast medium for MRI and injected following an 8-fold dilution at ratio of seven saline: One gadopentetate dimeglunmine. An 
Allegra 3.0T MRI Scanner (Siemens AG) was used for scanning, whilst an 8-channel head coil was used for collection. The fast spin echo (T2 weighted image turbo spin echo) was first used to obtain a conventional coronal T2-weighted image scan through the inner hear duct plane to exclude any lesions in the intracerebral and cerebellopontine angle areas. A three-dimensional (3D)-FLAIR sequence was then used to produce a high-resolution labyrinth scan and localization throughout the inner ear.

Multiplanar image reconstruction (Picture archiving and communication systems, PACS/RIS 3.1; Neusoft Medical Systems Co., Ltd.) was performed to obtain horizontal images for analysis. Following injection of the contrast reagent, the peri-lymphatic fluid was shown as the high-intensity zone whereas the membranous labyrinth was presented as the low-intensity zone on the 3D-FLAIR MRI original thin-layer image.

Subsequently, 3D reconstruction (Picture archiving and communication systems, PACS/RIS 3.1; Neusoft Medical Systems Co., Ltd.) was performed on the image at the post-processing stage to measure the area of endolymph space in the vestibulum and the total area of ipsilateral vestibulum auris. The following calculation was used to determine the ratio $(\mathrm{R})$ : $\mathrm{R}=$ low-intensity zone/(low-intensity zone + high-intensity zone) $\mathrm{x} 100 \%$. Using the following diagnostic criteria proposed by Nakashima et al (26), endolymphatic hydrops was diagnosed in cases of moderate hydrops and severe hydrops: i) Mild hydrops, $\mathrm{R}$ value $\leq 33.3 \%$; ii) moderate hydrops, $\mathrm{R}$ value $>33.3$ and $\leq 50.0 \%$; and iii) severe hydrops, $\mathrm{R}$ value $>50.0 \%$.

Vestibular-evoked myogenic potential (VEMP) test. A CHARTR Diagnostics System (model MCU-90; ICS Medical Corporation) was used for the VEMP (ICS Chartr EP 200; Natus Medical, Inc.) test (27). The recording electrode was first placed onto the skin surface at the midpoint of the sternocleidomastoid muscle, whilst the reference electrode was placed onto the upper part of sternum. The center of the forehead was grounded. The parameters used for the test were the following: i) Band-pass filtering, 50-3,000 Hz; ii) scanning time, $100 \mathrm{msec}$; iii) stacking fold, 150; iv) stimulation rate, $5.1 / \mathrm{sec}$; v) short pure tone frequency, $500 \mathrm{~Hz}$; vi) envelope, $2 \mathrm{msec}$ rise/decline; vii) plateau stage, $0 \mathrm{msec}$; and viii) alter polarity, stimulation intensity, 95 decibels $(\mathrm{dB})$ normal hearing level (dBnHL). During the examination, each individual was asked to raise the head by $30^{\circ}$ to tense up the sternocleidomastoid muscle at certain times but kept the head and neck still to record the VEMP latency, amplitude under bilateral short sound stimulation condition and to calculate the VEMP bilateral amplitude ratio and symmetry. A bilateral amplitude ratio $>1.61$ was considered to be abnormal.

Electrocochleography (EcochG) test. A CHARTR Diagnostics System (model MCU-90; ICS Medical Corporation) was also used for the EcochG test. A Life-Tech ear electrode model 8501 (Life-Tech) was used as a silver bead electrode, whilst the ICS Medical insert earphones $300 \mathrm{ohms}$ (ICS Medical Corporation) was applied as an insert earphone. This test was conducted in a sound-proof chamber. The silver bead electrode was placed underneath the posterior lower quadrant of the eardrum, whilst the reference electrode was placed at the ipsilateral earlobe. The contralateral earlobe was grounded. Test parameters were recorded as follows: i) Short sound stimulation, cycle $100 \mu \mathrm{sec}$; scanning time, $10 \mathrm{msec}$; band-pass filtering, 5-3,000 Hz; sparse wave/dense wave alter polarity, gain 50-100 k; stimulation rate, 11.1/sec; stacking fold, 1,000; and stimulation intensity, $90 \mathrm{dBnHL}$; ii) Short tone burst, envelope $2 \mathrm{msec}$ rise/decline; plateau stage, $10 \mathrm{msec}$; scanning time, $20 \mathrm{msec}$; band-pass filtering, 5-3,000 Hz; alter polarity, gain 50-100 k; stimulation rate, 11.1/sec, stacking fold, 1,000; and stimulation intensity, $90 \mathrm{dBnHL}$. The amplitude of summating potentia (SP) and Action potential and Compound action potential (AP) were measured with the initial baseline set as the standard, in $\mu \mathrm{V}$. SP/AP $\geq 0.4$ was considered to be abnormal.

Auditorybrainstemresponse (ABR).Using the SmartEPM010000 device (Intelligent Hearing Systems), click short sound stimulation was used to record the response threshold, wave I, III, V latency and interpeak latency in guinea pigs. The test is carried out in the room with sound insulation and electric shielding. The recording electrode was then placed subcutaneously on the top of the skull before the reference electrode was placed in the posterior region of the ipsilateral ear in guinea pigs. The tip of the nose is grounded. Earphone and hollow silicone tube were inserted and connected to the external auditory canal of guinea pigs. The mean and $2 \mathrm{X}$ standard deviation were used as the criterion for abnormity. Wave I, $\geq 1.82 \mathrm{msec}$; wave III, $\geq 4.14 \mathrm{msec}$; and wave $\mathrm{V}, \geq 6.02 \mathrm{msec}$ were considered as prolonged latency. The distortion product otoacoustic emission (OAE3.75, Intelligent Hearing Systems; https://www.medicalexpo.com.cn/prod/ intelligent-hearing-systems/product-79938-500100.html) was applied to record the response amplitude and signal-to-noise ratio.

ELISA. ELISA was used for detecting the concentrations of CIC (cat. no. JL11776; Shanghai Jianglai industrial, Ltd.), tumor necrosis factor- $\alpha$ (TNF- $\alpha$; cat. no. FT-P32761R; Shanghai Fantai Biotechnology Co., Ltd.) and heat shock protein 70 (HSP70; cat. no. FT-P32201R; Shanghai Fantai Biotechnology Co., Ltd.; https://www.hbzhan.com/st628355/ product_20565139.html) in the serum samples of patients with MD and those from healthy individuals according to the manufacturer's protocols. The concentration of isologous crude inner ear antigens (ICIEAg) was measured from $\sim 2 \mathrm{ml}$ serum of guinea pigs before euthanasia (the guinea pigs were euthanized immediately after blood collection) using a specially made ELISA kit (provided by Shanghai Fantai Biotechnology Co., Ltd.) which was constructed by Shanghai Fantai Biotechnology Co., Ltd. After the blood samples were collected, they were kept at room temperature for $15 \mathrm{~min}$ and then stored at $4^{\circ} \mathrm{C}$ until further use. The samples were then centrifuged at $1,200 \times \mathrm{g}$ for $30 \mathrm{~min}$ at $4^{\circ} \mathrm{C}$ before the supernatant was transferred into a new microcentrifuge tube and stored at $-20^{\circ} \mathrm{C}$ for subsequent use.

The standard was diluted serially five times, according to the manufacturer's protocols. Standard, blank and test sample wells were then constructed, using $50 \mu \mathrm{l}$ of the serially-diluted standard, no sample or enzyme-labeled reagent and $4 \mu \mathrm{l}$ standard diluent and $10 \mu \mathrm{l}$ test sample, respectively. 
After loading the samples, the plate was sealed then incubated at $37^{\circ} \mathrm{C}$ for $30 \mathrm{~min}$. The coated plate was then rinsed after the liquid was discarded and then spin-dried following which, $50 \mu \mathrm{l}$ enzyme-labeled reagent was added into the standard and test sample wells and incubated at $37^{\circ} \mathrm{C}$ for $30 \mathrm{~min}$. Subsequently, the plate was spin-dried for a second time, before $50 \mu \mathrm{l}$ developer $\mathrm{A}$ and $\mathrm{B}$ were added into each well and the plate was incubated at $37^{\circ} \mathrm{C}$ for $15 \mathrm{~min}$ after mixing.

Subsequently, $50 \mu 1$ stop buffer was added into each well, the blank well was zeroed and the absorbance [optical density (OD)] of each well was measured at $450 \mathrm{~nm}$ using a Varioskan LUX reader (Thermo Fisher Scientific, Inc.). The concentration of each sample was determined using a calibration curve, which was plotted based on the concentration and OD value of the standard samples.

Construction and processing of the autoimmune MD guinea pig model. The construction of the model refers to the previous research (28). The animal experiments were performed at the Specific Pathogen Free Animal Laboratory at the Inner Mongolia Medical University (Hohhot, China). A total of 45 male guinea pigs (purchased from GemPharmatech, Co., Ltd.; age, 5 months; weight, 600-900 g) were housed with four animals per cage at $20-25^{\circ} \mathrm{C}$ with $60 \%$ humidity in a quiet and well-ventilated environment with 12-h light/dark cycle., where food (containing vitamin C) and water were supplied freely. Animal health and behavior were monitored every day and body weights were assessed weekly over the course of the study.

ICIEAgs were obtained as follows: In total, five guinea pigs were decapitated under anesthesia (pentobarbital sodium $40 \mathrm{mg} / \mathrm{kg}$ was injected intraperitoneally) to obtain the inner ear tissues and to separate and place the membranous labyrinth in PBS for grinding, pulverizing and mixing. This solution was centrifuged using $15-\mathrm{cm}$ centrifuge tubes at $200 \mathrm{x}$ g at $4^{\circ} \mathrm{C}$ for $10 \mathrm{~min}$. The supernatant was collected to obtain the protein content following quantification using the bicinchoninic acid Protein Assay kit (Beyotime Institute of Biotechnology).

ICIEAg $(0.4 \mathrm{mg})$ and Freund's adjuvant $(0.2 \mathrm{ml})$ were injected in each animal for immunization, following which ICIEAg $(0.2 \mathrm{mg})$ and Freund's adjuvant $(0.2 \mathrm{ml})$ were injected 10 days later for two supplementary immunizations (fortified immunity), at an interval of 10 days. The guinea pigs were also tested under different $\mathrm{Hz} 1$ day before immunization. At total of 10 days after the last immunization, ICIEAg $(0.05 \mathrm{mg})$ and Freund's adjuvant $(0.025 \mathrm{ml})$ were used for local lymph sac immunization (local lymph sac immunization). The concentration of ICIEAg in immunity (ICIEAg immunization 10 days), fortified immunity (ICIEAg and Freund's adjuvant immunization 10 days after the immunity stage) and lymphatic sac immunity (ICIEAg and Freund's adjuvant immunization 10 days after the fortified immunity stage) groups was measured using ELISA. The hearing threshold of pre-immunization (before the ICIEAg immunization) and post-immunization (2 weeks after local lymph sac immunization) group at 4,8 and $16 \mathrm{kHz}$ was measured via ABR. In total each animal (30 animals) were immunized four times with three injections per immunization. In total, 10 guinea pigs were immunized with PBS as the control group.
A total of 30 guinea pigs were used for the further study and were randomly divided into the following three groups (10 guinea pigs per group): i) Group A, normal saline $(\mathrm{NaCl})$ group; ii) group B, dexamethasone group (intraperitoneal injection of dexamethasone, $2 \mathrm{mg} / \mathrm{d} / \mathrm{kg}$ ); and iii) group C, CTX group (intraperitoneal injection of CTX, $2.5 \mathrm{mg} / \mathrm{d} / \mathrm{kg}$ ). Guinea pigs were housed in individual cages, with ad libitum access to food and water and administered. After 2 weeks of raising, guinea pigs was treated with dexamethasone or CTX in different groups. Guinea pigs were anesthetized using an intraperitoneal injection of $40 \mathrm{mg} / \mathrm{kg}$ sodium pentobarbital. Under anesthesia, all guinea pigs were decapitated. ICIEAg concentration in the serum was also measured 2 weeks after being treated with dexamethasone or CTX in the three groups before euthanasia. Death was confirmed by the stopping of the heart and breathing rate, as well as the disappearance of the foot withdrawal reflex.

The present study was approved by the Animal Ethics Committee of Affiliated Hospital of Inner Mongolia Medical University (approval no. IMMU2018028; Hohhot, China). All related experiments were conducted in accordance with the 'Code for the Care and Use of Animals for Scientific Purposes' statement and under the principles of 3R (replacing, refining, and reducing) (29).

Total inner ear protein extraction and western blotting. Following intraperitoneal injection for anesthesia, the guinea pigs were decapitated to rapidly obtain the inner ear tissues and to remove the entire membranous labyrinth tissue under sterile conditions at $4^{\circ} \mathrm{C}$. The tissue was placed into PBS containing $40 \mathrm{~g} / 1$ phenylmethylsulfonyl fluoride, $10 \mathrm{mmol} / 1$ $\beta$-mercaptoethanol and $1 \% \mathrm{SDS}$, mixed at $4^{\circ} \mathrm{C}$, frozen and thawed for four cycles $\left(-20\right.$ to $\left.4^{\circ} \mathrm{C}\right)$ before being centrifuged at $12,000 \mathrm{x}$ g at $4^{\circ} \mathrm{C}$ for $20 \mathrm{~min}$. The concentration of the protein in the supernatant was then determined using bicinchoninic acid Protein Assay kit and separated using 10\% SDS-PAGE (40 $\mu \mathrm{g}$ protein per lane). The proteins were then transferred onto a PVDF membrane before being blocked for $1 \mathrm{~h}$ with $5 \%$ skimmed milk at $25^{\circ} \mathrm{C}$. After washing, the membrane was incubated at $4^{\circ} \mathrm{C}$ overnight with the following prediluted primary antibodies: Anti-HSP70 (1:1,000; cat. no. ab2787; Abcam) and anti- $\beta$-actin (1:2,000; cat. no. ab8226; Abcam). The membrane was then incubated with horseradish peroxidase (HRP)-conjugated secondary antibody $(1: 1,000$; cat. no. A0208; Beyotime Institute of Biotechnology) without agitation for $60 \mathrm{~min}$ at $20-25^{\circ} \mathrm{C}$. Signals were visualized using ECL reagents (EMD Millipore) and detected using an Amersham $^{\mathrm{TM}}$ Imager 680 (Cytiva).

Hematoxylin and eosin $(H \& E)$ staining. Following cardiac puncture and blood withdrawal from the guinea pigs under anesthesia, they were decapitated under anesthesia to rapidly harvest their inner ear tissues and for fixation in $4 \%$ paraformaldehyde for $24 \mathrm{~h}$ at $25^{\circ} \mathrm{C}$. EDTA-Na $(10 \mathrm{mM})$ was used for decalcification for $10-15$ days at $25^{\circ} \mathrm{C}$. Tissues were paraffin-embedded at $56^{\circ} \mathrm{C}$ and sliced at the central axis (10-mm thickness), followed by deparaffinization at $60^{\circ} \mathrm{C}$ for $10 \mathrm{~min}$, a $15 \mathrm{~min}$ immersion in xylene and rehydrated in a descending ethanol gradient. hematoxylin (5 min) and eosin (1 min) staining at $25^{\circ} \mathrm{C}$ and observed using an Olympus light microscope at $\mathrm{x} 400$ magnification (Olympus Corporation). 
Table I. Clinical characteristics of the patients with MD and healthy controls.

\begin{tabular}{|c|c|c|c|}
\hline Characteristics & Control $(\mathrm{n}=48)$ & $\operatorname{MD}(n=48)$ & P-value \\
\hline Mean age \pm SD (years) & $45.27 \pm 1.215$ & $43.79 \pm 1.347$ & $>0.05$ \\
\hline Sex, male, n $(\%)$ & $18(37.5)$ & $20(41.7)$ & $>0.05$ \\
\hline Mean weight \pm SD $(\mathrm{kg})$ & $67.04 \pm 1.006$ & $69.1 \pm 1.082$ & $>0.05$ \\
\hline \multicolumn{4}{|c|}{ Pure tone audiometry, n (\%) } \\
\hline Phase I & & $5(10.4)$ & \\
\hline Phase II & & $16(33.3)$ & \\
\hline Phase III & & $20(41.7)$ & \\
\hline Phase IV & & $7(14.6)$ & \\
\hline \multicolumn{4}{|c|}{ Endolymphatic hydrops, n (\%) } \\
\hline Light & & $10(20.8)$ & \\
\hline Medium & & $27(56.3)$ & \\
\hline Severe & & $11(22.9)$ & \\
\hline \multicolumn{4}{|l|}{ VEMP, n (\%) } \\
\hline Normal & & $20(41.7)$ & \\
\hline Abnormal & & $18(37.5)$ & \\
\hline Negative & & $10(20.8)$ & \\
\hline \multicolumn{4}{|l|}{ ECochG, n (\%) } \\
\hline Normal & & $19(39.6)$ & \\
\hline Abnormal & & $29(60.4)$ & \\
\hline
\end{tabular}

VEMP, vestibular evoked myogenic potential; ECochG, electrocochleography; MD, Meniere's disease.

Immunohistochemistry detection of CIC expression in the inner ear. The inner ear tissues were removed under anesthesia and the cochleae was separated. A hole was created at the cochlear tip to open the oval and round windows. At $4^{\circ} \mathrm{C}$, the cochlea was perfused with PBS containing $4 \%$ paraformaldehyde via the cochlear tip. The cochleae were then fixed in the same solution for $24 \mathrm{~h}$ at $25^{\circ} \mathrm{C}$ and rinsed with PBS before being placed into formic acid. In sodium formate solution, 1 week of decalcification was performed at room temperature, with the decalcifying solution replaced every day. After rinsing using PBS, the cochleae were removed and placed into a $30 \%$ sucrose solution overnight at $4^{\circ} \mathrm{C}$. After sedimentation, optimal cutting compound embedding (cat. no. 4583; Sakura Finetek Europe B.V.) was performed. The cryostat slicer moved in $10 \mu \mathrm{m}$ sections, in a parallel direction to the modiolus for continuous slicing at $-20^{\circ} \mathrm{C}$. After drying, CIC immunohistochemistry was performed.

The tissue sections were deparaffinized by a $15 \mathrm{~min}$ immersion in xylene. The sections were then rehydrated via sequential incubation in 100, 90 and 70\% ethanol. Samples were rinsed with PBS followed by distilled water and incubated for $30 \mathrm{~min}$ in $3 \% \mathrm{H}_{2} \mathrm{O}_{2}$ at $25^{\circ} \mathrm{C}$. Antigen retrieval (cat. no. P0085; Beyotime Institute of Biotechnology) was performed via microwave irradiation at $95^{\circ} \mathrm{C}$ for $10 \mathrm{~min}$. The sections were first permeabilized using $0.3 \%$ Triton $\mathrm{X}-100$ at $25^{\circ} \mathrm{C}$ for $5 \mathrm{~min}$ and then blocked with $10 \%$ rabbit serum (Beijing Solarbio Science \& Technology Co., Ltd.) at $37^{\circ} \mathrm{C}$ for $20 \mathrm{~min}$. Afterwards, sections were incubated with anti-C3 (1:1,000; cat. no. ab200999; Abcam) at $4^{\circ} \mathrm{C}$ overnight (an important component of CIC) $(30,31)$. The slides were washed four times in TBS/saponin and incubated with horseradish peroxidase-conjugated rabbit SignalStain ${ }^{\circledR}$ BoostIHCDetection Reagent (1:2,000, cat. no. 8114S; Cell Signaling Technology, Inc.) for $30 \mathrm{~min}$ at $25^{\circ} \mathrm{C}$ for $1 \mathrm{~h}$. The slides were incubated with a $0.5 \mathrm{mg} / \mathrm{ml} \mathrm{HRP}$ substrate solution $\left(\mathrm{DAB}+\mathrm{H}_{2} \mathrm{O}_{2}\right.$ prepared in distilled water) to expose the resulting peroxidase activity. Slides were washed four times in PBS and counterstained for $1 \mathrm{~min}$ with hematoxylin at $25^{\circ} \mathrm{C}$. The slides were sealed and observed under optical light microscopy at x40 magnification.

Statistical analysis. SPSS v19.0 (IBM Corp.) and GraphPad Prism v7 statistical software packages (Graphpad Software, Inc.) were used for statistical analyses. The measurement data are presented as the mean \pm SD. Student's t-test was used for between-group comparisons, whilst one-way ANOVA with Tukey's post hoc test was used for pairwise comparisons among multiple groups. Pearson's test was conducted to analyze the correlation between plasma and inner ear CIC concentration. The enumeration data were analyzed using $\chi^{2}$ test and linear correlation analysis. $\mathrm{P}<0.05$ was considered to indicate a statistically significant difference.

\section{Results}

Clinical information. There were a total of 48 patients with MD and 48 healthy control volunteers who were included in the present study. There were 18 males in the control group (37.5\%) and 20 males in the MD group (41.7\%). The mean weight of the patients was $67.04 \pm 1.01$ and $69.1 \pm 1.08 \mathrm{~kg}$ in control and MD groups, respectively. There was no significant difference in the age, sex distribution and weight distribution between the two groups (Table I). In addition, the clinical characteristics 
A
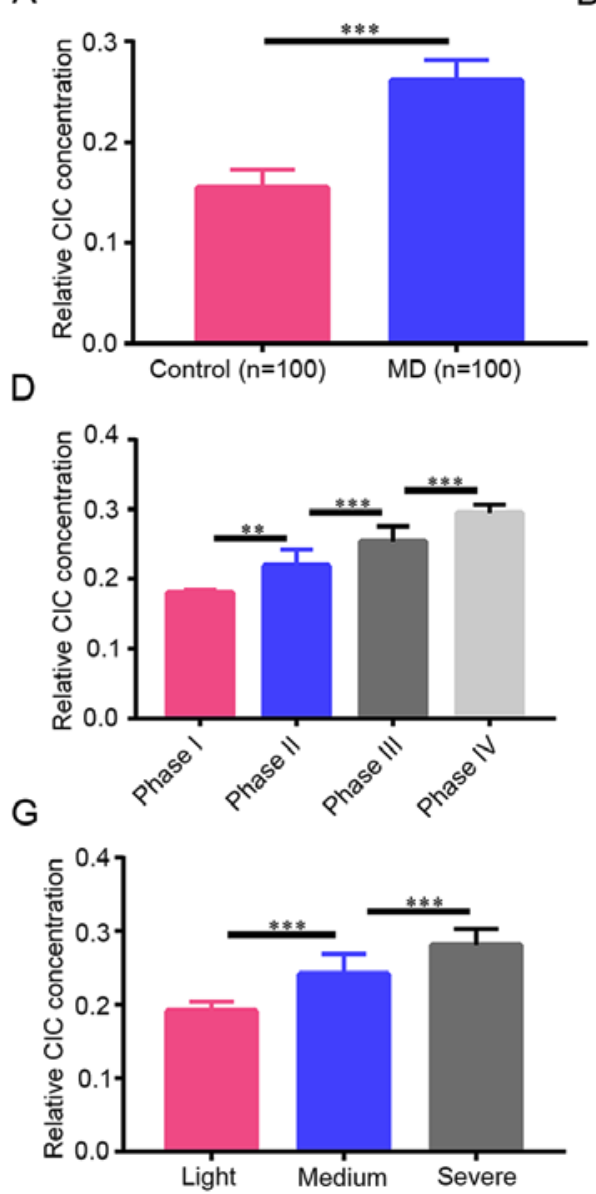

B

\section{E}
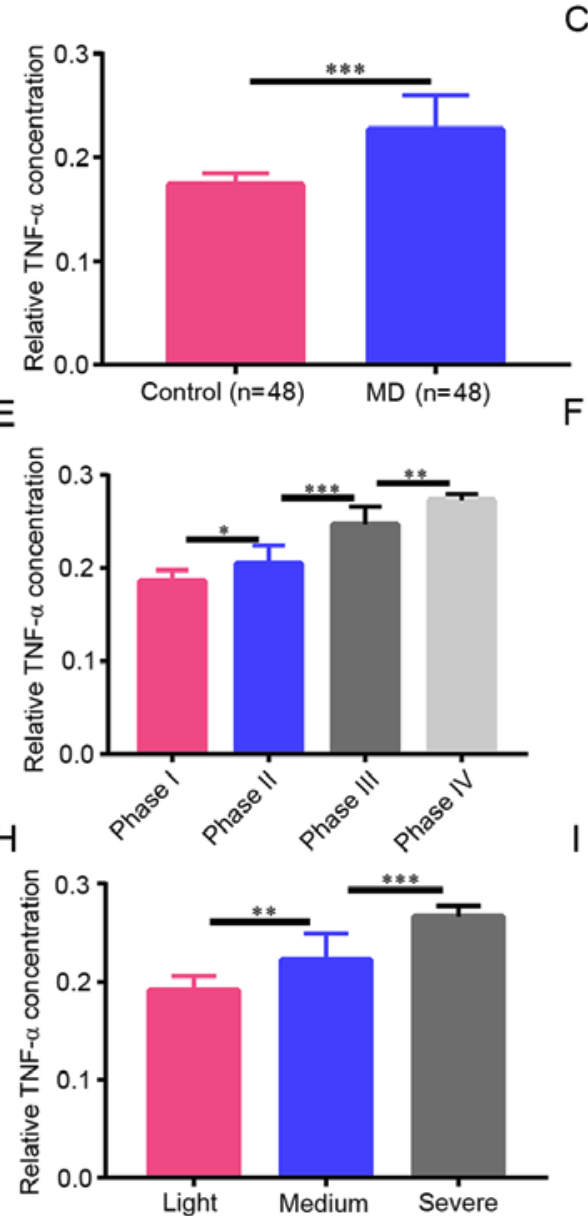

C

$\mathrm{F}$
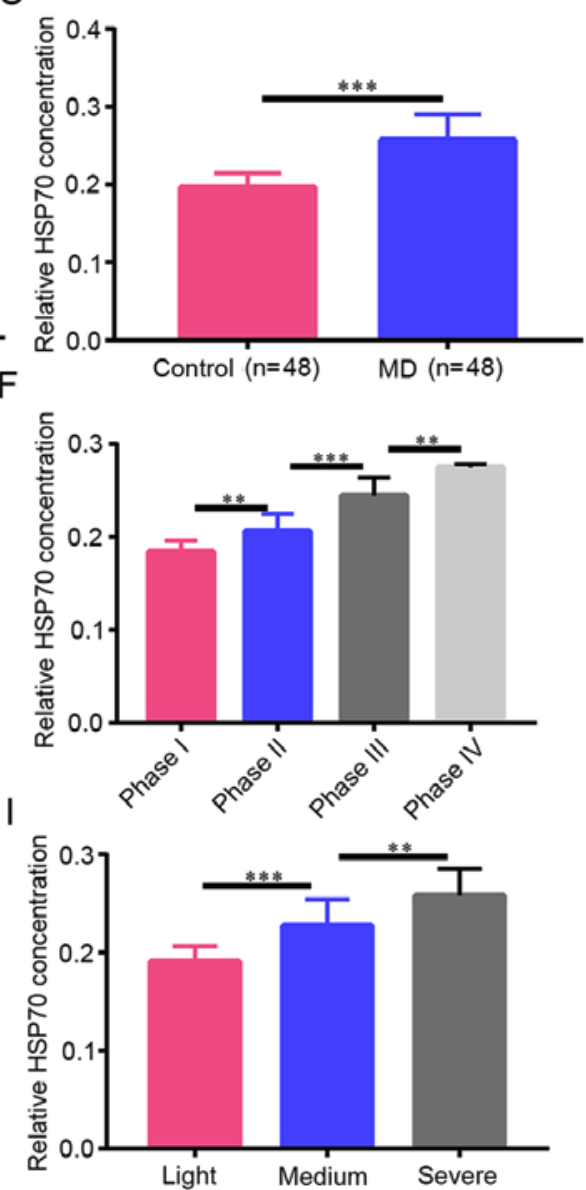

Figure 1. Expression of the immune parameters in the serum of patients with MD. ELISA was applied to detect the concentration of (A) CIC, (B) TNF- $\alpha$ and (C) HSP70 was upregulated in the serum of patients with MD compared with that in the control group. (D) CIC, (E) TNF- $\alpha$ and (F) HSP70 levels in the serum of patients with MD were increased in a manner that was dependent on the phases of pure tone audiometry tested by ELISA. The concentration of (G) CIC, (H) TNF- $\alpha$ and (I) HSP70 in the serum of patients with MD was increased in a manner that was dependent on the severity of endolymphatic hydrops via ELISA. ${ }^{*} \mathrm{P}<0.05,{ }^{* *} \mathrm{P}<0.01$ and ${ }^{* * *} \mathrm{P}<0.001$. CIC, circulating immune complex; TNF, tumor necrosis factor, HSP70, heat shock protein 70 ; MD, Meniere's disease.

and stage of the patients with MD were analyzed (Table I). Pure tone audiometry was investigated in the patients with MD and the results found that $10.4(n=5), 33.3(n=16), 41.7(n=20)$ and $14.6 \%(n=7)$ of patients had stage I, II, III and IV, respectively (Table I). MRI was subsequently used to assess the degree of endolymphatic hydrops in the patients with MD and the results revealed that $20.8(n=10), 56.3(n=27)$ and $22.9 \%(n=11)$ of patients had mild, moderate and severe endolymphatic hydrops, respectively (Table I). The VEMP test showed that normal, abnormal and negative VEMP was observed in $41.7(n=20), 37.5(n=18)$ and $20.8 \%(n=10)$ of patients, respectively (Table I). Finally, the results of the ECochG test revealed that $39.6(n=19)$ and $60.4 \%(n=29)$ of patients had normal and abnormal results, respectively (Table I).

Concentration of immune parameters in the serum from patients with $M D$. To investigate the association between MD and the immune response, the concentration of CIC, TNF- $\alpha$ and HSP70 in the serum of patients with MD was investigated. The results showed that the concentration of CIC, TNF- $\alpha$ and HSP70 was significantly increased in the serum of patients with MD compared with that in the control group, suggesting that the occurrence and development of MD was associated with the immune response (Fig. 1A-C). Subsequently, the association between the concentration of CIC, TNF- $\alpha$ and HSP70 and the clinical characteristics of patients with MD, was also analyzed. The results indicated that the phase of pure tone audiometry was positively associated with the concentration of CIC, TNF- $\alpha$ and HSP70 in the serum of patients with MD (Fig. 1D-F). In addition, the concentrations of CIC, TNF- $\alpha$ and HSP70 in the serum of patients with MD were also positively associated with the the severity of endolymphatic hydrops (Fig. 1G-I). Taken together, CIC in the serum of patients with MD were significantly increased compared with that in the control group, demonstrating that immune factors can potentially be important for the development of MD. Further analysis of the results indicated that immune indices CIC, TNF- $\alpha$ and HSP70 are positively associated with the clinical stages and severity of MD, suggesting that they can be applied as potential diagnostic markers of MD.

CIC expression in the autoimmune MD guinea pig model. To further identify the potential value of CIC for the diagnosis and treatment of MD, a guinea pig MD model was established 
A

E

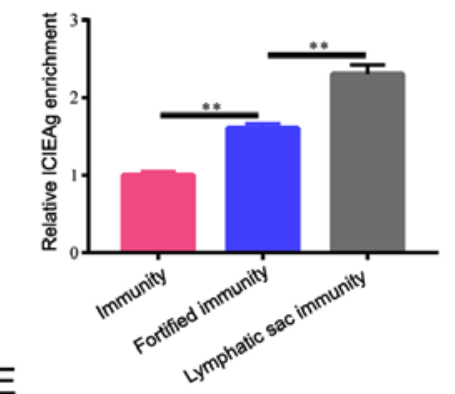

B

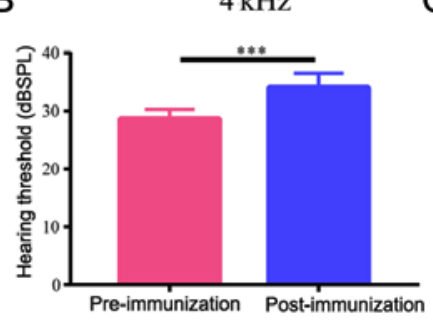

C

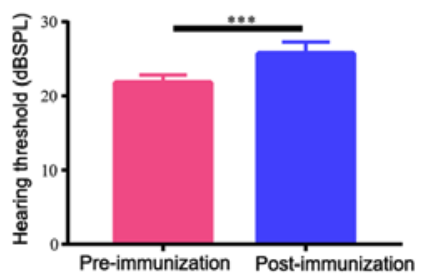

D

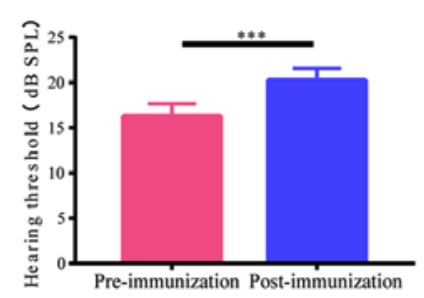

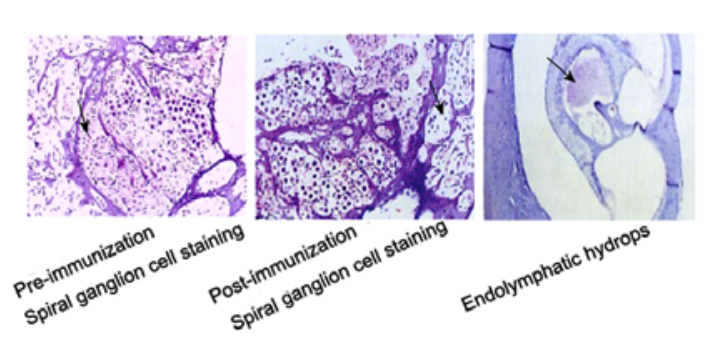

$\mathrm{F}$

G
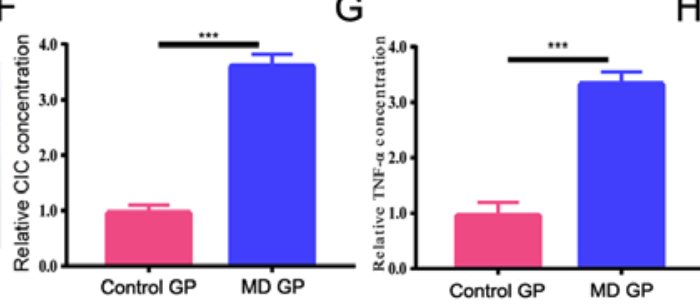

$\mathrm{H}$

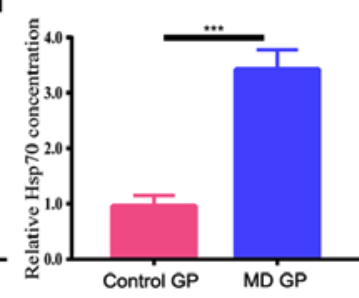

Figure 2. CIC expression level in the autoimmune MD guinea pig model. (A) ELISA was conducted to detect the ICIEAg concentration in guinea pigs in early immunization, after systemic booster immunization and local lymph sac immunization was gradually increased after each stage of immunization. The auditory brainstem response threshold at (B) 4,(C) 8 and (D) $16 \mathrm{kHz}$ threshold was notably higher 2 weeks after immunization compared with that before immunization through auditory brainstem response. (E) Hematoxylin and eosin staining results indicated that 2 weeks after immunization, spiral ganglions were fewer in number in guinea pigs with extensive endolymphatic hydrops. Magnification x400. (F) CIC, (G) TNF- $\alpha$ and (H) HSP70 concentration in serum was significantly increased in guinea pigs with MD compared with that in the control group via ELISA. ${ }^{* *} \mathrm{P}<0.01$ and ${ }^{* * *} \mathrm{P}<0.001$. GP, guinea pigs; $\mathrm{dBSPL}$, Decibel Sound Pressure Level; CIC, circulating immune complex; TNF, tumor necrosis factor; HSP70, heat shock protein 70; MD, Meniere's disease.

using autoimmunity via injection with ICIEAg isolated from other guinea pigs. To determine if the model had been successfully established, the concentration of ICIEAg in guinea pigs during the early immunization stage, after systemic booster immunization and local lymph sac immunization was detected. The results of ELISA showed that after each stage of immunization, ICIEAg concentration in guinea pigs was significantly increased compared with that at the previous stage (Fig. 2A), suggesting effective immunization. In total, 1 day before immunization and 2 weeks after the final ICIEAg + freund's adjuvant, the ABR threshold at 4,8 and $16 \mathrm{kHz}$ was measured, where the results revealed that at all three frequencies, the threshold post-immunization was significantly higher compared with that before immunization (Fig. 2B-D). $\mathrm{H} \& \mathrm{E}$ staining results indicated that at 2 weeks post-immunization, spiral ganglions were notably fewer in number in guinea pigs with endolymphatic hydrops clearly observed compared with that in the pre-immunization group (Fig. 2E), suggesting successful construction of the MD guinea pig model. Subsequently, CIC expression level in the control and MD guinea pigs (2 weeks after the final immunization) were determined and it was found that CIC expression was significantly increased in the serum of MD guinea pigs compared with that in the control group (Fig. 2F), which further implicate the potentially important roles of CIC during the course of MD. In addition, TNF- $\alpha$ and HSP70 concentrations in the MD guinea pigs were significantly higher compared with that in the control group (guinea pigs immunized with PBS; Fig. $2 \mathrm{G}$ and $\mathrm{H}$ ).

CTX suppresses $M D$ progression by reducing CIC generation. To investigate the effects of CTX treatment on
$\mathrm{MD}, \mathrm{MD}$ guinea pigs were randomly divided into the $\mathrm{NaCl}$, dexamethasone and CTX groups, with 10 guinea pigs in each group, with treatment beginning 2 weeks after the final immunization. At 1 (Fig. 3A-C) and 2 weeks (Fig. 3D-F) post-treatment, changes in the ABR threshold were detected at 4,8 and $16 \mathrm{kHz}$ in each group. The results revealed that the threshold was the highest in the $\mathrm{NaCl}$ group, followed by the dexamethasone and CTX groups, with the differences among the three groups significant (Fig. 1A-F), suggesting that CTX is more effective compared with that in dexamethasone. ICIEAg concentration in the serum was next measured 2 weeks after treatment in the three groups and it was found that the concentration of ICIEAg in the dexamethasone group was significantly lower compared with that in the $\mathrm{NaCl}$ group, whilst the ICIEAg concentration in the CTX group was significantly decreased compared with that in the dexamethasone group (Fig. 3G). Subsequently, CIC expression was detected in the serum samples of each group after 2 weeks treatment, where the highest CIC expression was found in the $\mathrm{NaCl}$ group, followed by the dexamethasone and CTX groups (Fig. 3H), suggesting that CTX exerted its roles by reducing CIC generation. Subsequently, ELISA was used to detect the CIC deposition in the inner ear tissue of each group after 2 weeks treatment and the results revealed that CIC expression showed the same trend as that exhibited by CIC levels in the serum (Fig. 3I), suggesting that CTX also reduced CIC deposition in the inner ear tissues. Finally, Pearson's correlation analysis was used to analyze the correlation between CIC expression in the serum and inner ear tissues of each group, where the results indicated that CIC expression in the serum was positively correlated with that in the inner ear tissue in all three treatment groups tested 
A

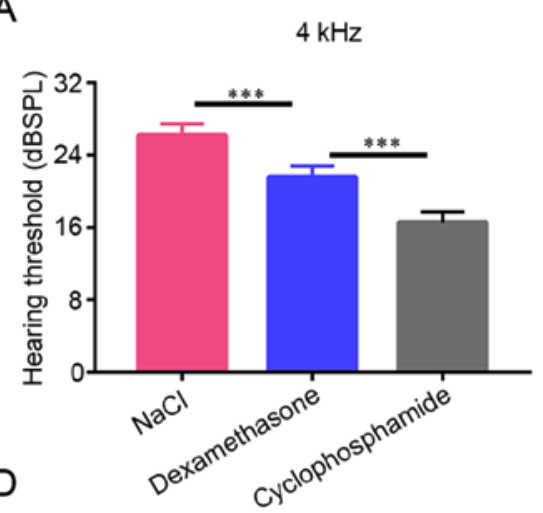

$4 \mathrm{kHz}$
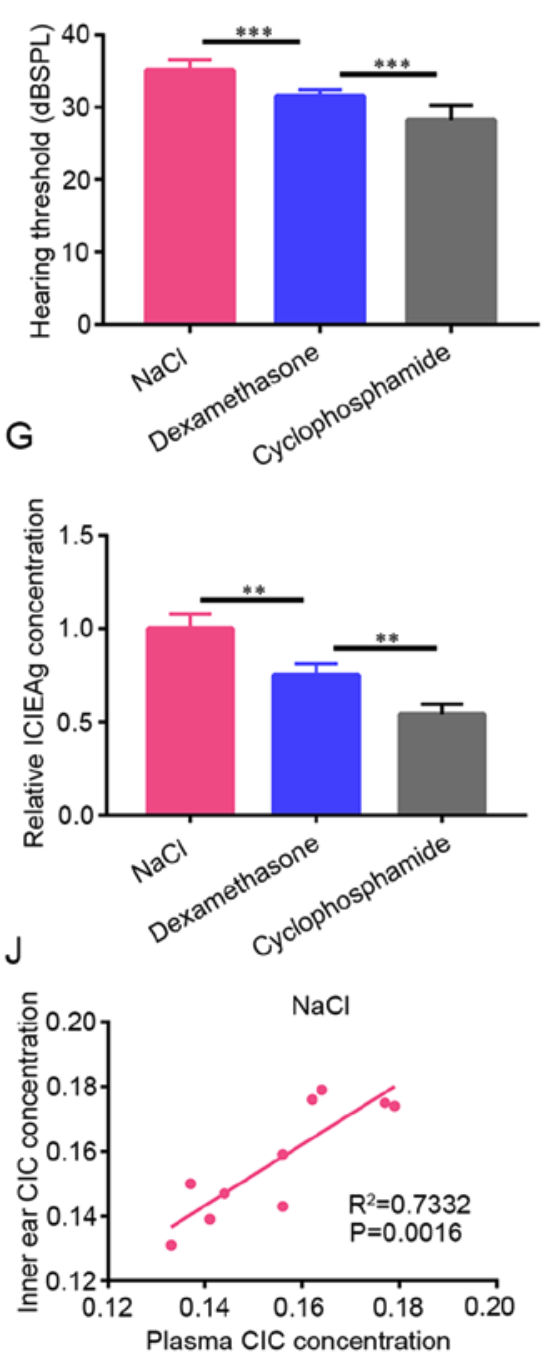

B

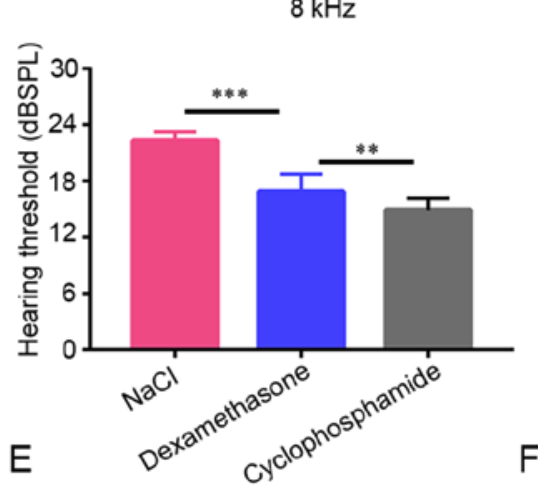

$8 \mathrm{kHz}$
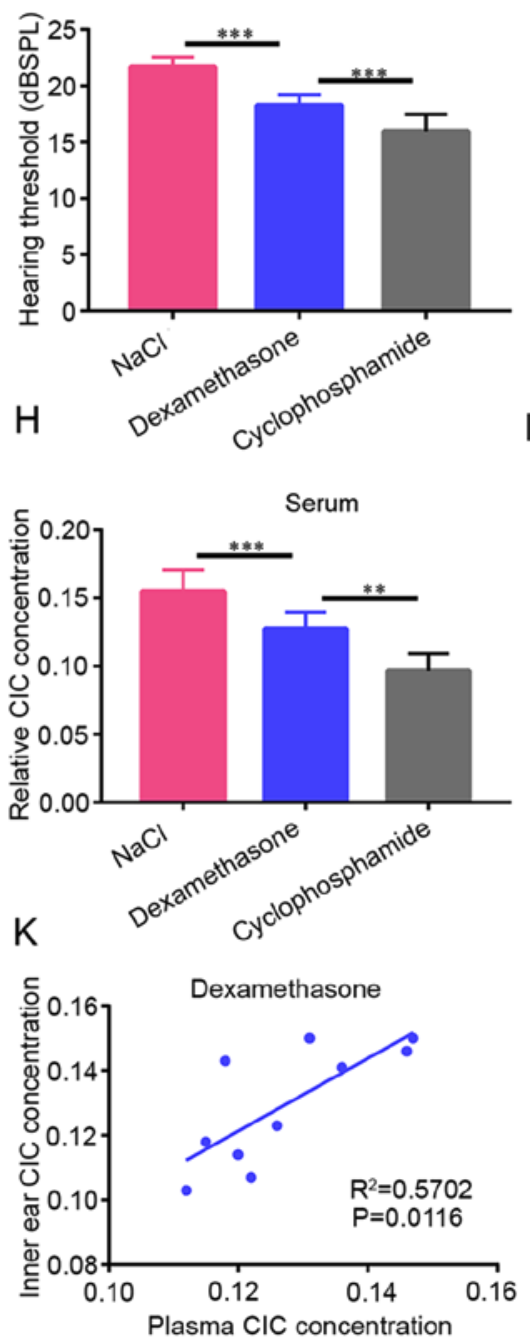

C

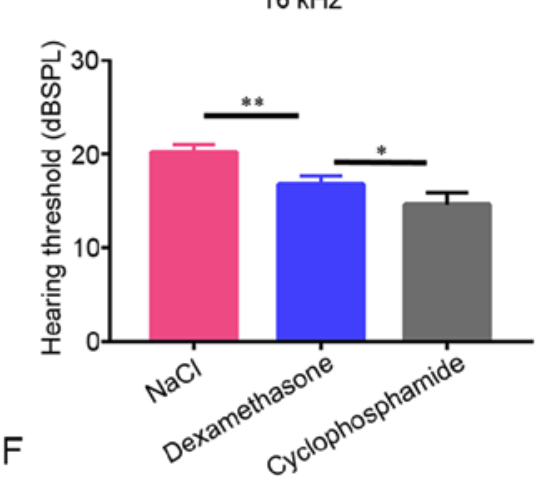

$16 \mathrm{kHz}$
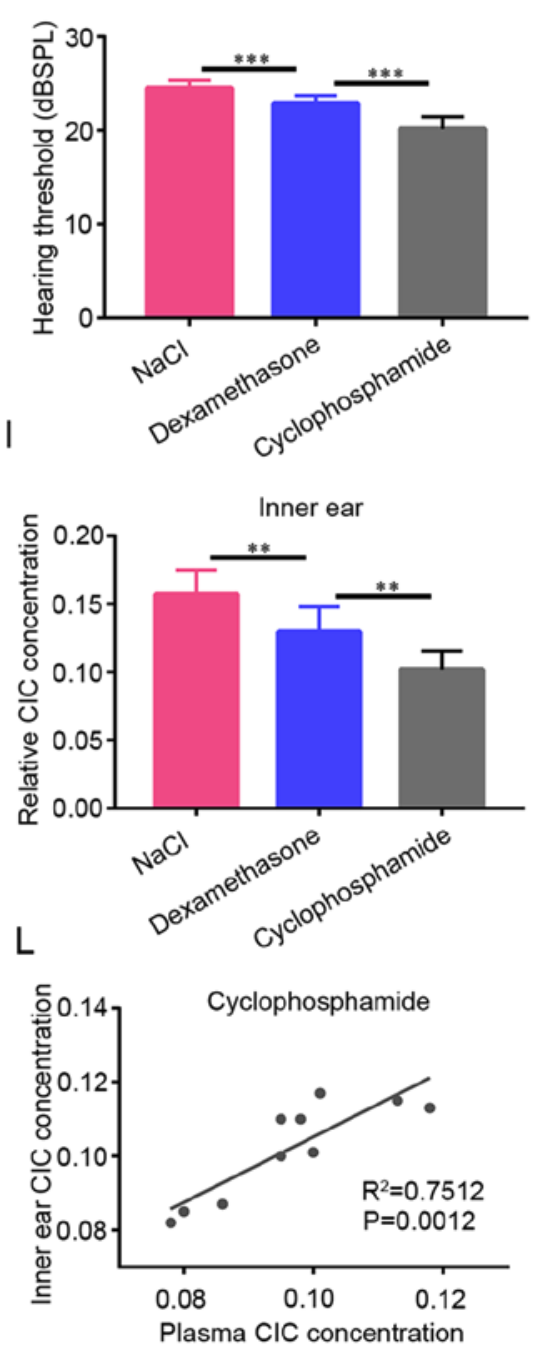

Figure 3. CTX suppresses MD progression by reducing CIC generation. A total of 1-week post-treatment, the ABR threshold at (A) 4, (B) 8 and (C) $16 \mathrm{kHz}$ was the highest in the $\mathrm{NaCl}$ group, followed by the dexamethasone group and then the CTX group. After 2 weeks of treatment, the CTX group exhibited the lowest ABR threshold at (D) 4, (E) 8 and (F) $16 \mathrm{kHz}$ and guinea pigs in the $\mathrm{NaCl}$ group had the highest ABR threshold. (G) ICIEAg expression in the dexamethasone group was significantly lower compared with that in the $\mathrm{NaCl}$ group, whilst ICIEAg expression in the CTX group was significantly decreased compared with that in the dexamethasone group tested by ELISA. The CIC concentration in the (H) serum or (I) inner ear tissues was highest in the NaCl group, followed by the dexamethasone group and then the CTX group detected by ELISA. (J-L) Pearson's correlation analysis showed the CIC concentration in the serum was positively correlated with that in the inner ear tissue in the $(\mathrm{J}) \mathrm{NaCl},(\mathrm{K}) \mathrm{CTX}$ and $(\mathrm{L})$ Dexamethasone groups. ${ }^{*} \mathrm{P}<0.05,{ }^{* * *} \mathrm{P}<0.01$ and ${ }^{* * * *} \mathrm{P}<0.001 . \mathrm{CTX}$, cyclophosphamide; MD, Meniere's disease; ICIEAg, isologous crude inner ear antigens; ABR, auditory brainstem response.

(Fig. 3J-L). This suggest that CIC deposition in the inner ear tissue could be found by detecting CIC expression in the circulation, rendering CIC expression to be potentially useful as an index for evaluating the degree of MD. CTX was also found to suppress MD progression more effectively than dexamethasone by reducing CIC generation. 
A

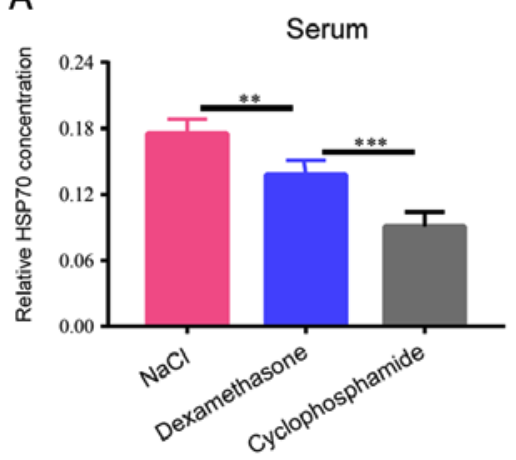

C

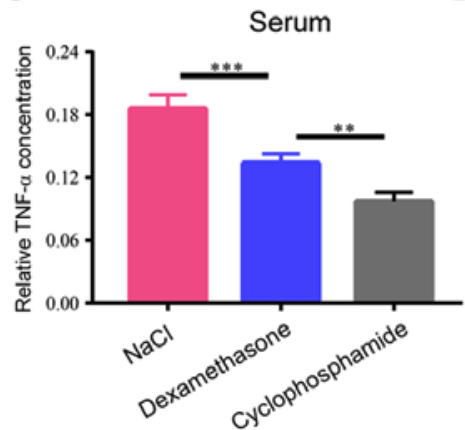

B

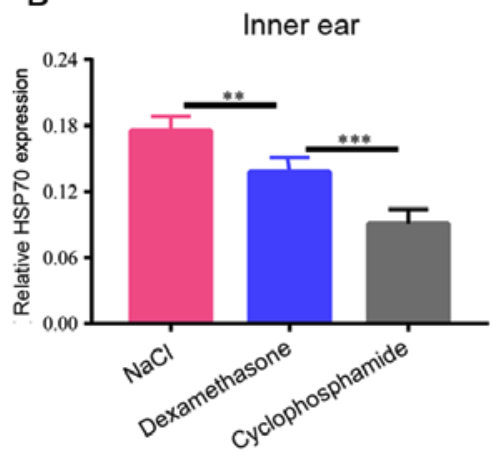

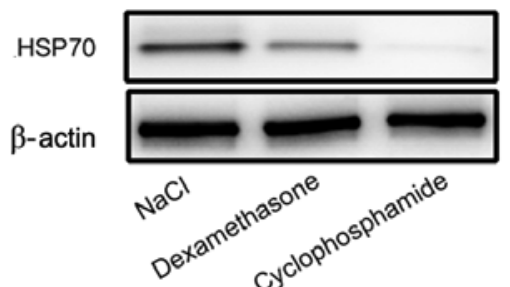

E
D

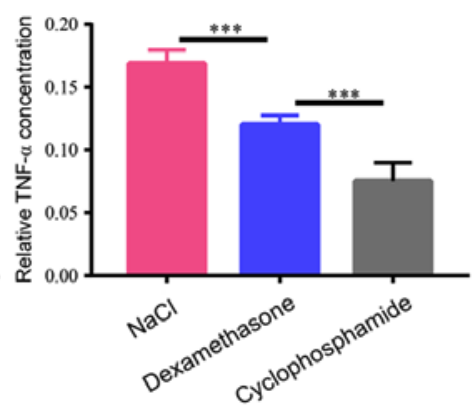

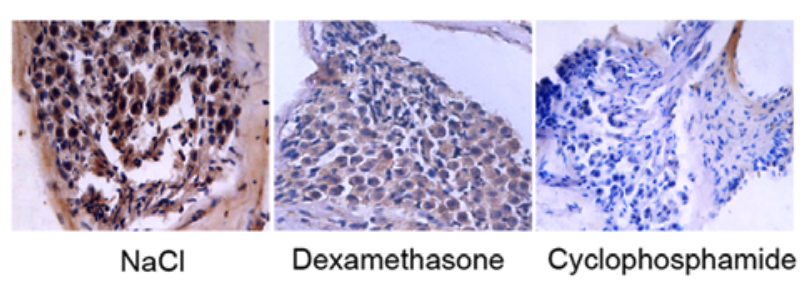

Figure 4. Effects of CTX on HSP70 and TNF- $\alpha$ concentration levels. (A) ELISA revealed that HSP70 concentration in the circulation of the dexamethasone group was significantly lower compared with that in the $\mathrm{NaCl}$ group, whilst HSP70 concentration in the CTX group was significantly reduced compared with that in the dexamethasone group. (B) Western blotting showed that HSP70 protein expression in the inner ear of the dexamethasone group was significantly lower compared with that in the $\mathrm{NaCl}$ group, whilst HSP70 concentration in the CTX group was significantly reduced compared with that in the dexamethasone group. ELISA showed that the TNF- $\alpha$ concentration in the (C) serum or (D) the inner ear tissues was also decreased in the NaCI, dexamethasone compared with that in the $\mathrm{NaCl}$ group, whilst TNF- $\alpha$ concentration was lower in the CTX group compared with that in the dexamethasone group. (E) Immunohistochemical staining showed that the highest CIC deposition was in the $\mathrm{NaCl}$ group, followed by the dexamethasone group and then the CTX group. Magnification, $\mathrm{x} 400$. ${ }^{* *} \mathrm{P}<0.01$ and ${ }^{* * *} \mathrm{P}<0.001$. CIC, circulating immune complex; TNF, tumor necrosis factor; HSP70, heat shock protein 70; CTX, cyclophosphamide.

Effects of CTX on HSP70 and TNF- $\alpha$ concentration. Considering that CTX may reduce the generation of CIC, it was hypothesized whether the same role could be found with the immune indices HSP70 and TNF- $\alpha$. The results of the ELISA showed that HSP70 concentration in the circulation of the dexamethasone group was significantly lower compared with that in the $\mathrm{NaCl}$ group, whereas HSP70 concentration in the CTX group was significantly reduced compared with that in the dexamethasone group (Fig. 4A). After harvesting the inner ear tissue from each group, western blot analysis was used to detect the protein expression level of HSP70 in the inner ear tissue of each group and the results revealed the same trend of HSP70 expression in the inner ear tissue, with HSP60 expression being the lowest in the CTX group (Fig. 4B). ELISA was then used to measure TNF- $\alpha$ concentration in the inner ear tissue and serum samples of each group and the results revealed that the lowest $\mathrm{TNF}-\alpha$ concentrations were found in the CTX group (Fig. 4C and D). This suggest that in addition to the reduction of CIC generation, CTX also suppressed HSP70 and TNF- $\alpha$ expression. Finally, inner ear tissues were collected from each group for immunohistochemical staining and the results found the most $\mathrm{CIC}$ deposition in the $\mathrm{NaCl}$ group, followed by the dexamethasone and CTX groups (Fig. 4E). As shown by the aforementioned results, CTX exerted specific effects on MD in manners that were more effective compared with those exerted by dexamethasone, possibly by inhibiting the autoimmune response.

\section{Discussion}

The typical histopathological manifestation of MD is endolymphatic hydrops, which may involve the cochlea, vestibulum and semicircular canal to damage the cochlear and vestibular functions (32). However, the association between the symptoms and corresponding pathological manifestations induced by these symptoms remain unclear. Histopathological changes in patients with MD cannot be assessed directly during the course of the disease in a clinical setting. Therefore, the present study aimed to provide specific clinical data to further elucidate the factors underlying the occurrence and development of MD by detecting CIC concentration in the circulation. The present study enrolled 48 patients with MD and 48 control volunteers, who were not significantly different in terms of their age, sex or weight.

Previous studies $(27,33)$ showed that VEMP was weakened or could not be induced in $35-54 \%$ patients with MD. In the present study, VEMP was weakened or could not be induced in $58.3 \%$ of patients, which was consistent with these previous studies aforementioned. It has been reported that ECochG changes are one of the most typical audiological manifestations of endolymphatic hydrops (1). ECochG manifestation in patients with MD is represented by higher SP/AP ratios, which are associated with higher SP or lower AP (34). This is induced by excessive lymph fluids, resulting in the displacement of the basilar membrane (35). However, this examination lacks sensitivity (35). The positive rate of abnormal ECochG was only 
found to be $60.4 \%$ in the present study. Nevertheless, similar to the results in the present study, Helling et al (36) performed ECochG in 334 patients with MD and found abnormal results in $~ 56.3 \%$ of patients. This was probably due to the higher SP or lower AP caused by multiple factors, reducing the specificity of ECochG. High resolution MRI provides new opportunities for the diagnosis of MD and enables a clear display of the endolymphatic hydrops (37). In the present study, MRI was used to analyze endolymphatic hydrops in 48 patients, where more moderate hydrops $(56.3 \%)$ were found, with less mild and severe hydrops (20.8 and $22.9 \%$, respectively).

CIC is a substance formed by the combination of antigens and corresponding antibodies that immune complexes bind to during the complement pathway and other immunoreactive substances (38). They deposit onto the vascular wall, resulting in tissue injury and vasculitis (39). If immune complexes are detected in the circulating blood, they would be known as CICs. If CIC deposits in the stria vascularis and endolymphatic sac, endolymphatic hydrops may be induced accordingly (18). Savastano et al (40) previously analyzed the roles of non-specific serological immunity tests in determining the immunopathology of patients with MD and found that CIC was significantly increased in serum, leading to the hypothesis that CIC determination can be used as a prognosis index to monitor the clinical development of MD. In the present study, ELISA was utilized to detect CIC concentration in the patients with MD and in the control group. The results revealed notably higher CIC levels in patients with MD and that CIC was increased with the progression of clinical stage and the severity of endolymphatic hydrops. This suggest that CIC could have a certain diagnostic value for the occurrence and development of MD, which can be used as a biomarker. In addition, TNF- $\alpha$ and HSP70 concentrations showed the same trend in patients with MD, indicating that immune factors are also important during the course of MD.

Considering existing studies of collagen type II-induced autoimmune ear disease, Matsuoka et al (41) found that the guinea pig endolymphatic hydrops model could be induced by directly injecting the collagen type II peptide monoclonal antibody into the scala tympani, with a success rate of $\sim 100 \%$. This method could be used for the study of autoimmune MD (40). To further investigate the association between CIC and $\mathrm{MD}$, in vivo experiments were performed in the present study by constructing an autoimmune MD guinea pig model. After local immunization using ICIEAg in systemically sensitized guinea pigs, guinea pigs developed significant hearing loss. The results of immunohistochemistry revealed markedly less spiral ganglion cells in the guinea pig model group and endolymphatic hydrops in the inner ear, suggesting that the autoimmune MD guinea pig model was successfully constructed. Using ELISA, CIC concentration was significantly increased in the MD guinea pig model compared with that in the control group. In addition, immune parameters TNF- $\alpha$ and HSP70 were also notably increased, further demonstrating an association between immune factors and MD onset.

There is currently no consensus and effective method for the treatment of MD. A previous study has found that betahistine may promote blood flow into the cochlea (42) that may be used to reduce the degree and frequency of vertigo in patients with MD. However, evidence of its effectiveness remains controversial. Bodmer et al (43) treated patients with MD by drip transfusion of gentamicin into the tympanum and found that long-term vertigo was controlled effectively. However, aminoglycosides conferred vestibulotoxicity for the treatment of MD and therefore are not commonly used in a clinical setting (36). A previous study has found that injecting steroid hormones into the tympanum was effective for MD treatment (44). However, in another study it observed that the injection of dexamethasone into the tympanum was only a temporary alternative treatment option for MD, where endolymphatic hydrops was temporarily reduced 1 month after treatment but returned to the initial value 1 year later (45). Recent studies have observed that immunosuppressants were effective for MD $(46,47)$. A study previously found that CTX may notably improve hearing in patients with autoimmune inner ear disease (48). Kilpatrick et al (49) observed that a small dose of oral methotrexate was effective and safe for the treatment of immune-mediated bilateral MD. However, there are only a few studies investigating the efficacy of immunosuppressants for the treatment of immune-mediated MD. In addition, no report exists on the mechanism of action of CTX for immune-mediated MD. Therefore, further investigation is required.

In the present study, MD guinea pigs were first randomly divided into the $\mathrm{NaCl}$, dexamethasone and CTX groups. At 7 and 14 days post-administration, the ABR threshold was measured in each group. The frequency was statistically significantly different between the dexamethasone and $\mathrm{NaCl}$ groups. Local dexamethasone treatment was effective in the treatment of autoimmune MD. However, CTX was more effective for the treatment of autoimmune MD compared with that in dexamethasone, providing a theoretical basis for guiding clinical medication for MD. Subsequently, the expression of $\mathrm{CIC}$ and other immune parameters in the circulation and the inner ear tissue was detected, where it was found that CTX significantly reduced the expression of CIC, TNF- $\alpha$ and HSP70 compared with that in the dexamethasone group. In addition, immunohistochemistry results confirmed lower CIC deposition in the inner ear tissue of the CTX group, suggesting that CTX can inhibit MD development by reducing CIC expression.

In conclusion, the present study provided novel strategies for the treatment of early MD, which is difficult to diagnose using clinical screening and data. In addition, the present study also provides evidence for the potential value of serum CIC concentration for the diagnosis, staging and prognosis assessment of autoimmune MD. To address the amibiguity of MD treatment, the present study investigated the mechanism of action and efficacy of CTX for the treatment of autoimmune MD using guinea pig models, to demonstrate the immunological mechanism of MD and provide an experimental foundation for the clinical promotion of drug therapy. These data can provide a potential development strategy for the early diagnosis and drug therapy of MD.

\section{Acknowledgements}

Not applicable. 


\section{Funding}

The present study was supported by the Natural Science Foundation of Inner Mongolia Autonomous Region (grant no. 2019MS08123) and Inner Mongolia Medical University Youth Innovation Fund project (grant no. YKD2017QNCX064).

\section{Availability of data and materials}

The datasets used and/or analyzed during the present study are available from the corresponding author on reasonable request.

\section{Authors' contributions}

SZ designed the study. SZ and YG conducted the guinea pig experiments. YL, BW, WG and QX conducted data analysis and interpretation of data. SZ and YG authenticate the data in this study. All authors read and approved the final revision of the manuscript.

\section{Ethics approval and consent to participate}

Animal use and care were performed in accordance with the Guide for the Care and Use of Laboratory Animals published by the US National Institutes of Health. This study was approved by the Animal Ethics Committee of Affiliated Hospital of Inner Mongolia Medical University (grant no. IMMU2018028). Written informed consent was provided from the patients and the healthy controls Animal experiments were performed in the SPF Animal Laboratory at Inner Mongolia Medical University.

\section{Patient consent for publication}

Not applicable.

\section{Competing interests}

The authors declare that they have no competing interests.

\section{References}

1. AnftD,JamaliY,ScholzGandMrowinskiD:Electrocochleography and phase audiometry in diagnosis of Meniere disease. HNO 49 : 102-108, 2001 (In German).

2. Sun Y, Zhang D, Sun G, Lv Y, Li Y, Li X, Song Y, Li J, Fan Z and Wang H: RNA-sequencing study of peripheral blood mononuclear cells in sporadic Meniere's disease patients: Possible contribution of immunologic dysfunction to the development of this disorder. Clin Exp Immunol 192: 33-45, 2018.

3. Pai I, Mendis S, Murdin L, Touska P and Connor S: Magnetic resonance imaging of Meniere's disease: Early clinical experience in a UK centre. J Laryngol Otol 134: 302-310, 2020.

4. Hallpike CS and Cairns H: Observations on the pathology of Meniere's syndrome: (Section of otology). Proc R Soc Med 31: 1317-1336, 1938.

5. Caulley L, Quimby A, Karsh J, Ahrari A, Tse D and Kontorinis G: Autoimmune arthritis in Meniere's disease: A systematic review of the literature. Semin Arthritis Rheum 48: 141-147, 2018.

6. Greco A, Gallo A, Fusconi M, Marinelli C, Macri GF and de Vincentiis M: Meniere's disease might be an autoimmune condition? Autoimmun Rev 11: 731-738, 2012.
7. Cooper MW and Kaylie DM: Is endolymphatic sac surgery beneficial for Meniere's disease? Laryngoscope 130: 2738-2739, 2020.

8. Mathews J, Rao S and Kumar BN: Autoimmune sensorineural hearing loss: Is it still a clinical diagnosis? J Laryngol Otol 117 212-214, 2003

9. Dornhoffer JL, Waner M, Arenberg IK and Montague D: Immunoperoxidase study of the endolymphatic sac in Meniere's disease. Laryngoscope 103: 1027-1034, 1993.

10. Gazquez I, Soto-Varela A, Aran I, Santos S, Batuecas A, Trinidad G, Perez-Garrigues H, Gonzalez-Oller C, Acosta L and Lopez-Escamez JA: High prevalence of systemic autoimmune diseases in patients with Meniere's disease. PLoS One 6: e26759, 2011.

11. Harada T, Matsunaga T, Hong K and Inoue K: Endolymphatic hydrops and III type allergic reaction. Acta Otolaryngol 97: 450-459, 1984

12. Lopez-Escamez JA, Saenz-Lopez P, Gazquez I, Moreno A, Gonzalez-OllerC,Soto-Varela A, Santos S,Aran I,Perez-Garrigues H, Ibañez A and Lopez-Nevot MA: Polymorphisms of CD16A and $\mathrm{CD} 32 \mathrm{Fc} \gamma$ receptors and circulating immune complexes in Meniere's disease: A case-control study. BMC Med Genet 12: 2, 2011.

13. Derebery MJ, Rao VS, Siglock TJ,Linthicum FH and Nelson RA: Meniere's disease: An immune complex-mediated illness? Laryngoscope 101: 225-229, 9991.

14. Hsu L, Zhu XN and Zhao YS: Immunoglobulin E and circulating immune complexes in endolymphatic hydrops. Ann Otol Rhinol Laryngol 99: 535-538, 1990.

15. Kim SH, Kim JY, Lee HJ, Gi M, Kim BG and Choi JY: Autoimmunity as a candidate for the etiopathogenesis of Meniere's disease: Detection of autoimmune reactions and diagnostic biomarker candidate. PLoS One 9: e111039, 2014.

16. Zheng $\mathrm{N}$ and Lu Y: Targeting the IL-9 pathway in cancer immunotherapy. Hum Vaccin Immunother 16: 2333-2340, 2020

17. Oh S, Lee JH, Kwack K and Choi SW: Natural killer cell therapy: A new treatment paradigm for solid tumors. Cancers (Basel) 11: 1534,2019

18. Derebery MJ and Berliner KI: Allergy and its relation to Meniere's disease. Otolaryngol Clin North Am 43: 1047-1058, 2010.

19. Di Berardino F and Zanetti D: Delayed immunomodulatory effect of cow milk-free diet in Meniere's Disease. J Am Coll Nutr 37: 149-153, 2018.

20. Kim J and Chan JJ: Cyclophosphamide in dermatology. Australas J Dermatol 58: 5-17, 2017.

21. Berd D, Mastrangelo MJ, Engstrom PF, Paul A and Maguire H: Augmentation of the human immune response by cyclophosphamide. Cancer Res 42: 4862-4866, 1982.

22. Emadi A, Jones RJ and Brodsky RA: Cyclophosphamide and cancer: Golden anniversary. Nat Rev Clin Oncol 6: 638-647, 2009.

23. Tsuchida Y,Harada M, Shoda H, Goto A, Suzuki N, Murashima A, Osuga $\mathrm{Y}$ and Fujio K: Fertility preservation in patients receiving gonadotoxic therapies for systemic autoimmune diseases in Japan. Mod Rheumatol: Jan 18, 2021 (Epub ahead of print). doi: $10.1080 / 14397595.2020 .1856020$

24. Editorial Board of Chinese Journal of Otorhinolaryngology Head and Neck Surgery and Society of Otorhinolaryngology Head and Neck Surgery Chinese Medical Association: Guideline of diagnosis and treatment of Meniere disease (2017). Zhonghua Er Bi Yan Hou Tou Jing Wai Ke Za Zhi 52: 167-172, 2017 (In Chinese).

25. Kim DY, Kwon J, Kim JY, Cha HS, Kim YW, Kim IY and Im CH: New method for pure-tone audiometry using electrooculogram: A proof-of-concept study. Sensors (Basel) 18: 3651, 2018.

26. Nakashima T, Naganawa S, Pyykko I, Gibson WP, Sone M, Nakata $S$ and Teranishi M: Grading of endolymphatic hydrops using magnetic resonance imaging. Acta Otolaryngol Suppl 560: $5-8,2009$..

27. Welgampola MS and Colebatch JG: Characteristics and clinical applications of vestibular-evoked myogenic potentials. Neurology 64: 1682-1688, 2005.

28. Lu L, Tan CQ, Cui YG, Ding GP, Ju XB, Li YJ and Cai WJ: Analysis of the main components of inner ear antigens inducing autoimmune Meniere's disease in guinea pigs. Zhonghua Er Bi Yan Hou Tou Jing Wai Ke Za Zhi 43: 596-600, 2008 (In Chinese).

29. Perry M: Revised australian code of practice for the care and use of animals for scientific purposes. Aust Vet J 76: 286, 1998.

30. Kraaij T, Nilsson SC, van Kooten C, Okroj M, Blom AM and Teng YO: Measuring plasma C4D to monitor immune complexes in lupus nephritis. Lupus Sci Med 6: e000326, 2019. 
31. Gupta S, Lau K, Harding CO, Shepherd G, Boyer R, Atkinson JP, Knight V, Olbertz J, Larimore K, Gu Z, et al: Association of immune response with efficacy and safety outcomes in adults with phenylketonuria administered pegvaliase in phase 3 clinical trials. EBioMedicine 37: 366-373, 2018.

32. Wright T: Meniere's disease. BMJ Clin Evid 2015: 0505, 2015.

33. Young $\mathrm{YH}, \mathrm{Wu} \mathrm{CC}$ and $\mathrm{Wu} \mathrm{CH}$ : Augmentation of vestibular evoked myogenic potentials: An indication for distended saccular hydrops. Laryngoscope 112: 509-512, 2002.

34. Mao Z, Liu L, Peng L, Zhou L and Liu A: Electrocochleography in the diagnosis of Meniere's disease. Lin Chung Er Bi Yan Hou Tou Jing Wai Ke Za Zhi 28: 964-967, 2014 (In Chinese).

35. Hornibrook J: Tone burst electrocochleography for the diagnosis of clinically certain Meniere's disease. Front Neurosci 11: 301, 2017.

36. Helling K, Schonfeld U and Clarke AH: Treatment of Meniere's disease by low-dosage intratympanic gentamicin application: Effect on otolith function. Laryngoscope 117: 2244-2250, 2007.

37. Gurkov R, Flatz W, Louza J, Strupp M, Ertl-Wagner B and Krause E: In vivo visualized endolymphatic hydrops and inner ear functions in patients with electrocochleographically confirmed Meniere's disease. Otol Neurotol 33: 1040-1045, 2012.

38. Tamai S: Physiological and pathological aspects of circulating immune complex. Nihon Rinsho 68 (Suppl 6): S105-S109, 2010 (In Japanese).

39. Salvidio G and Andres G: Immune deposits and immune complex disease. Clin Exp Rheumatol 4: 281-288, 1986.

40. Savastano M, Giacomelli L and Marioni G: Non-specific immunological determinations in Meniere's disease: Any role in clinical practice? Eur Arch Otorhinolaryngol 264: 15-19, 2007.

41. Matsuoka H, Kwon SS, Yazawa Y, Barbieri M and Yoo TJ: Induction of endolymphatic hydrops by directly infused monoclonal antibody against type II collagen CB11 peptide. Ann Otol Rhinol Laryngol 111: 587-592, 2002.
42. Ihler F, Bertlich M, Sharaf K, Strieth S, Strupp M and Canis M: Betahistine exerts a dose-dependent effect on cochlear stria vascularis blood flow in guinea pigs in vivo. PLoS One 7: e39086, 2012.

43. Bodmer D, Morong S, Stewart C, Alexander A, Chen JM and Nedzelski JM: Long-term vertigo control in patients after intratympanic gentamicin instillation for Meniere's disease. Otol Neurotol 28: 1140-1144, 2007.

44. Ren H, Yin T, Lu Y, Kong W and Ren J: Intratympanic dexamethasone injections for refractory Meniere' s disease. Int J Clin Exp Med 8: 6016-6023, 2015.

45. Martin-Sanz E, Esteban-Sanchez J, Rodriganez-Riesco L and Sanz-Fernandez R: Transitory effect on endolymphatic hydrops of the intratympanic steroids for Meniere's disease. Laryngoscope 125: 1183-1188, 2015.

46. Peneda JF, Lima NB, Monteiro F, Silva JV, Gama R and Conde A: Immune-mediated inner ear disease: Diagnostic and therapeutic approaches. Acta Otorrinolaringol Esp 70: 97-104, 2019.

47. Guo SY, Zhang Y and Liu B: Recent immunology research of Meniere's disease. Zhonghua Er Bi Yan Hou Tou Jing Wai Ke Za Zhi 53: 953-956, 2018 (In Chinese).

48. Ciorba A, Corazzi V, Bianchini C, Aimoni C, Pelucchi S, Skarżyński PH and Hatzopoulos S: Autoimmune inner ear disease (AIED): A diagnostic challenge. Int J Immunopathol Pharmacol 32: 2058738418808680, 2018.

49. Kilpatrick JK, Sismanis A, Spencer RF and Wise CM: Low-dose oral methotrexate management of patients with bilateral Meniere's disease. Ear Nose Throat J 79: 82-83, 86-88, 91-92, 2000 .

This work is licensed under a Creative Commons Attribution-NonCommercial-NoDerivatives 4.0 International (CC BY-NC-ND 4.0) License. 